\title{
Missionspresse im Dilemma zwischen Kirche und Medien, Auftrag und Auflage
}

\author{
Von Marcel Bauer
}

„Im Anfang war das Wort und das Wort war bei Gott“. So beginnt bekanntlich das Johannes-Evangelium. Ohne eine Exegese der Heiligen Schrift wagen zu wollen, ist anzumerken, daß der Evangelist Johannes - als intellektuelle Nachhut der drei Synoptiker Matthäus, Markus und Lukas - wohl das feinste Gespür für die Bedeutung des „Wortes“ entwickelt hat. Untersucht man heute den geistigen Standort, den das gesprochene und geschriebene Wort in unserer säkularisierten Gesellschaft einnimmt, drängt sich rasch der Eindruck auf, daß das Wort wohl überall vorhanden ist außer bei Gott.

Das Christentum ist anders als der Hinduismus oder der Buddhismus eine Religion des Buches. Die christliche Botschaft ist schriftlich fixiert, also abrufbar, ablesbar, absehbar. Anders als bei den beiden anderen heiligen Büchern, dem Koran und der Hebräischen Bibel des Judentums, ist die göttliche Offenbarung im Neuen Testament nicht das diktierte Wort Gottes, sondern sie bedient sich menschlicher Mittelsmänner, eben der Evangelisten. Diese vier Chronisten würde man heute mit dem etwas ominösen Wort Journalisten umschreiben. Auch der Apostel Paulus, dieser geniale Reisende in Sachen Evangelisation, machte das Wort zu seinem wichtigsten Instrument. Nicht von ungefähr hat Papst Johannes Paul I. während seines kurzen Pontifikats mit einem Bonmot für Aufregung gesorgt, als er meinte, der Heilige Paulus wäre heute wohl Chefredakteur einer großen Tageszeitung. In der Tat würde der Apostel Paulus, dieser talentierte Polemiker und „grand reporter“ seiner Zeit, auch heute noch im „Bericht aus Bonn“ oder im „Auslandsjournal“ gute Figur machen.

Nicht erst seit der Erfindung der Buchdruckerkunst hat die Kirche das gesprochene, geschriebene oder gespielte Wort zur Verbreitung ihrer Botschaft genutzt. Die Reformation und - als Reaktion darauf - die Gegenreformation haben sich in breiter Front der Medien bedient. Um so wunderlicher mutet es an, daß im Verhältnis von Kirche und Massenmedien, diesen alten verbündeten Schwestern der Vergangenheit, heute eher Mißtrauen und Vorurteile vorherrschen. Trotz päpstlicher Appelle und amtlicher Schmeicheleien hat sich innerhalb der Kirche immer mehr der Eindruck verfestigt, daß die Medien ein Werkzeug des Bösen seien. Die schmerzlichen Erfahrungen, die die Kirche oft mit den Massenmedien gemacht hat, haben etliche ihrer Vertreter dazu verleitet, jeden Kontakt mit der Presse zu meiden, ihr ihre Glaubwürdigkeit weitgehend abzusprechen und sogar ihre Nutzung für die Verkündigung in Frage zu stellen.

\section{Der Bildschirm bleibt auf Distanz}

In der Tat: Schlägt man heute eine Tageszeitung auf oder schaltet die Abendnachrichten des Fernsehens ein, kommen die Kirchen entweder überhaupt nicht oder nur am Rande vor. Diese meßbare Distanz zu den Kirchen ist in den privaten Printmedien und in den

Marcel Bauer hat Soziologie und Geschichte in Löwen und Berlin studiert. Der gebürtige Belgier ist Buch- und Filmautor. Seit 1975 leitet er die Pressestelle des Internationalen Katholischen Missionswerks MISSIO in Aachen. 
öffentlich-rechtlichen Rundfunk- und Fernsehanstalten unterschiedlich gelagert. Da das Gros der deutschen Zeitungs- und Zeitschriftenverlage konservativ ausgerichtet ist, darf man theoretisch in den Printmedien noch eine größere Resonanz und Rezipienz für kirchliche Themen erwarten. Schon programmatisch ist jedoch die Kirchenferne in den elektronischen Medien, was um so besorgniserregender ist, als daß nach einer jüngeren Umfrage $80 \%$ aller deutschen Jugendlichen ihre Informationsbedürfnisse ausschließlich durch das Fernsehen gedeckt sehen.

Diese Indifferenz der elektronischen Medien für kirchliche Themen mutet um so grotesker an, als die Väter des Rundfunk-Rahmengesetzes in den einzelnen Staatsverträgen der Kirche ausdrücklich ein Mitspracherecht in der Programmgestaltung einräumten. Neben den politischen Parteien und den großen Sozialverbänden sind die Kirchen nominell für die Ausgewogenheit des Programms und die paritätische Besetzung der Programmpositionen mitverantwortlich. Aber anders als etwa die Gewerkschaften, die im Laufe der Jahrzehnte in den öffentlich-rechtlichen Anstalten fast ein Proporzrecht erkämpft haben, wurden die Kirchen fast ohne Gegenwehr ins Dritte Programm oder auf die hohen kirchlichen Festtage abgeschoben.

Heute bemüht sich die Kirche nach Kräften, verlorene Positionen wieder wettzumachen und - etwa in den Rahmenverhandlungen mit dem privaten Fernsehen - auf Achtung ihres garantierten Status zu pochen. Schlimmer als der offene Antiklerikalismus, der hin und wieder in den Anstalten aufflackert, aber durch die kirchliche Repräsentanz in den Gremien immer unter Kontrolle blieb, ist das offenkundige Totschweigen von kirchlichen Anliegen. Dies muß einerseits der Kirche selbst angekreidet werden, die es zumindest in der Frühphase völlig an eigener Planung und Initiative im Medienbereich fehlen ließ.

Die kirchliche Vakanz liegt aber letzten Endes am Desinteresse, wenn nicht am stillen Boykott religiöser Themen durch verantwortliche Redakteure: Nach einer repräsentativen Umfrage unter Journalisten in Hörfunk und Fernsehen bezeichnen sich nur noch drei Prozent der hauptamtlichen Redakteure als aktive Mitglieder ihrer Kirchen. 20 bis 30 Prozent stufen sich als „laue Christen“ ein. Überdurchschnittlich hoch ist der Anteil der sogenannten Agnostiker: Etwa 50 Prozent aller Journalisten im öffentlich-rechtlichen Bereich geben an, keiner christlichen Konfession anzugehören. Diese Zahlen sind ein Menetekel.

Die Bedeutung des Fernsehens kann in einer Zivilisation des laufenden Bildes gar nicht hoch genug veranschlagt werden: Der Einflu $\beta$ des Fernsehens auf die breite Mehrheit unserer Bevölkerung wird nicht nur durch die Einschaltquoten der ausgestrahlten Sendungen wirksam. Das Fernsehen ist längst auch zum „Trendsetter“ überhaupt, auch für die nicht-elektronischen Medien geworden. Auch die Printmedien sind heute gezwungen, die Themen aufzugreifen, auf die Kontroversen und Konflikte einzugehen, die ihnen vom Fernsehen vorgegeben werden. In der Regel wird die Frankfurter Allgemeine Zeitung in ihrer Morgenausgabe die Fernseh-Debatte vom Vortag kommentieren. Nur im Ausnahmefall wird die Tagesschau in ihren Spätnachrichten aus einem Interview der Neuen Osnabrücker Zeitung zitieren. Partiell kann es aber zu Allianzen konkurrierender Medien kommen, wenn sich den Redaktionen gemeinsame weltanschauliche oder politische Ziele anbieten. Wenn auch einige Skandale das Ansehen der Hamburger Troika von Stern, Spiegel und Panorama angekratzt haben, wird ihre Zugkraft besonders bei der Propagierung ethischer „Alternativen“(Abtreibung, Euthanasie et cetera) immer wieder wirksam. 
Zu guter Letzt doch noch ein Wort zur vertraglich garantierten kirchlichen Vertretung in den elektronischen Medien. Der sogenannte Kirchenfunk ist ohne Verschuldung der zuständigen Redakteure zu einem Hinterstübchen im Riesenressort Kultur verkommen. Angesichts der Sendemassen, die diese zahlenmäßig schwach besetzten Redaktionen dank Staatsvertrag zu bewältigen haben, verdienen die Arbeitsleistung und das Engagement der Redakteure Respekt. Dies um so mehr, da sich diese kirchlichen Ableger in ihren Häusern häufig der Häme ihrer säkularisierten Umwelt ausgesetzt sehen. Die Kritik, die sie zusätzlich vom kirchlichen Amt wegen ihrer Programmgestaltung erfahren, fördert nicht sonderlich ihre kirchliche Bindung. So kann leicht der Eindruck entstehen, den Kirchenfunkredaktionen gehe es eher um Vermittlung politischer oder gesellschaftlicher Themen als um religiöse Erbauung oder christliche Motivation.

\section{Konservativ heißt noch nicht kirchlich}

Wie oben erwähnt, ist die „Säkularisierung“ in den Printmedien weniger artikuliert. Aber auch hier sollte eine konservative Herausgeberschaft nicht über die Orientierungslosigkeit der Redaktionen hinwegtäuschen. Die Säkularisierung hat auch hier tiefe Spuren hinterlassen. Um ein Beispiel zu nehmen: Die Scheidungsrate liegt derzeit in der Bundesrepublik bei 30 Prozent auf 100 geschlossene Ehen. Bei Männern und Frauen, die als Berufsangabe Journalist angeben, liegt sie weitaus höher: bei über 70 Prozent. Es ist jedoch eine Binsenwahrheit, daß die privaten Konflikte sich auch auf die Weltanschauung, zumindest aber auf die Loyalitäten auswirken. Die Generation der Altgedienten, die der Kirche die Stange hielten, stirbt aus - sowohl in den Gewerkschaften als auch in den Medien, ohne daß neue Lobbyisten nachwachsen.

Im zeitlichen Wettkampf mit den elektronischen Medien sind die Printmedien wegen ihrer umständlichen Herstellung und langwierigen Zustellung unterlegen. Sie haben sich daher mehr auf das Kommentieren und Reflektieren der Nachrichten verlagert, wobei sie - wie schon erwähnt - von den Vorgaben der elektronischen Medien abhängig bleiben. Die Rezipienz für kirchliche Themen in den privaten Printmedien ist zweifellos größer als in den öffentlich-rechtlichen Anstalten. Dennoch ist offenkundig, daß auch dort eher die Randthemen der Religion interessieren als ihre inhaltlichen Aussagen. Dies bedeutet beispielsweise für die kirchlichen Hilfswerke, daß sie mit einer um so gröBeren Übernahme ihrer Materialien in den Medien rechnen können, je weniger ihre Kirchlichkeit (inhaltlich oder imagemäßig) sichtbar wird: Zur kirchlichen Entwicklungshilfe oder zum Katastropheneinsatz kirchlicher Organisationen haben säkulare Medien eben leichter Zugang, während die Förderung oder Vernachlässigung der Missionshilfe durch die Medien unmittelbar vom Glaubensschwund oder vom Glaubenswachstum abhängt. Dies nötigt kirchliche Werke wie Adveniat oder MISSIO, stärker auf eher sekundäre Folgen der Evangelisation in der Dritten Welt hinzuweisen, etwa auf die wachsende Sensibilität für Menschenrechte und soziale Gerechtigkeit, auf moralische Erneuerung oder gar wirtschaftliche Dynamik, die sich in der Regel im Gefolge der Christianisierung einstellen.

Es wäre ein Trugschluß, aus der konservativen Grundhaltung der meisten großen Zeitungsverlage automatisch ein Wohlwollen für kirchliche Anliegen vorauszusetzen. Gerade in Bezug auf die Dritte Welt können die Fronten durcheinandergeraten, wie das 
Beispiel der Misereor-Aktion „Südliches Afrika“ belegt. Aber auch bei innenpolitischen Themen wie Asylanten-Frage, Wahlrecht für Gastarbeiter oder Waffengeschäfte mit der Dritten Welt sind die Karten gemischt.

In der Regel ist davon auszugehen, daß die Medien für die Kirche nur dann zur Verfügung stehen, wenn sie ihnen gesellschaftlich relevante Themen im säkularen Sinn anbietet. Viel stärker als die elektronischen Medien, deren Finanzierung zwangsweise über Gebühren erfolgt, stehen die Printmedien ständig im wirtschaftlichen Konkurrenzkampf. Während früher die hohen Abonnentenzahlen Zeitungen und Zeitschriften mittelfristig absicherten, wird heute die Existenz etlicher Publikationen jede Woche neu am Kiosk entschieden. Von der verkauften Auflage hängt unmittelbar das Anzeigenvolumen ab. Selbst bei Tageszeitungen werden 66 Prozent der Kosten durch Anzeigen gedeckt. Bei Illustrierten und Magazinen ist der Werbeanteil noch bedeutend höher zu veranschlagen. Der Kampf um den Kiosk wird von den Millionenblättern marktschreierisch und mit Millionenaufwand betrieben, so daß kirchliche Publikationen keine Chancen haben, dort zu landen. Von der gesamten katholischen Presse, die immerhin eine Auflage von 10 Millionen Exemplaren hat, sind im freien Verkauf nur noch eine Wochenzeitung und eine halbmonatliche Illustrierte, etwa vier Prozent der Gesamtauflage, vertreten.

\section{Abschied von kostspieligen Experimenten}

Der Deutschen Bischofskonferenz ist die Gefahr, daß die Kirche vom Medienmarkt verdrängt werden kann, frühzeitig bewußt geworden. Eine erste Reaktion war in den sechziger Jahren der Zusammenschluß regionaler Kirchenblätter zu großen Bistumszeitungen, auch wenn die redaktionellen Rahmenbedingungen bis heute zu wünschen übrig lassen. Ein zweiter Ansatz war seinerzeit die Gründung der Wochenzeitung „Publik“, die ein unrühmliches Ende nahm. Danach hat sich das Interesse der kirchlichen Verantwortlichen den elektronischen Medien zugewandt. Als Ende der siebziger Jahre das Schlagwort von den neuen Medien auftauchte, scheute die Bischofskonferenz keine Kosten, um in das Kabelpilotprojekt von Ludwigshafen mit einem eigenen Fernsehprogramm einzusteigen und sich an den Bildschirmtext-Experimenten der Bundespost von Hamburg bis Düsseldorf zu beteiligen. Während ein immenser technischer Aufwand von kirchlicher Seite betrieben wurde, wurde am Personal gespart, so daß das Scheitern beider Experimente eigentlich absehbar war. Zum Jahreswechsel hat man sich von beiden Projekten in aller Stille verabschiedet.

Anders als im europäischen Umfeld, wo die katholische Kirche etwa in Belgien, Luxemburg, den Niederlanden, Frankreich, Spanien und Italien über eine starke konfessionelle Presse in freier Trägerschaft verfügt, ist die Kirche in der Bundesrepublik - sieht man einmal von der Würzburger Tagespost, die im Zwei-Tage-Rhythmus erscheint, ab - im wesentlichen auf kirchliche Publikationen angewiesen. Die kirchliche Presse ist vom strukturellen Wandel der Printmedien in den späten fünfziger Jahren kaum berührt worden. Es ist ihr nicht gelungen, vom Image eines kirchlichen Mitteilungsblattes, das mit religiöser Erbauungsliteratur und harmloser Unterhaltung spärlich angereichert ist, loszukommen. Der Gesichtsverlust der kirchlichen Presse ist so weit fortgeschritten, daß sie selbst von ihren Herausgebern oft nicht mehr für fähig erachtet wird, das kirchliche Anliegen in der Öffentlichkeit zu vertreten. Einige Organe haben es daher vorgezogen, sich in hohe intellektuelle Regionen zurückzuziehen, so daß sie jeden Anspruch, 
eine breite Öffentlichkeit zu beeinflussen, von selbst aufgegeben haben. Mit dem Bezug einer Kirchenzeitung wird oft nur noch eine kirchliche Bindung demonstriert. Auflagenmäßig ist die kirchliche Presse immer noch ein Gigant, wenn auch die Frage berechtigt ist, inwieweit Auflage und effektive Leserschaft übereinstimmen. Umfragen zufolge kommen bei herkömmlichen Illustrierten und Zeitschriften mehrere Leser auf ein Exemplar. Ohne polemisch zu sein, wage ich zu behaupten, daß es bei der kirchlichen Presse eher umgekehrt ist, insofern auf einen Leser gleich mehrere Publikationen kommen.

Da, wo der Versuch gewagt wurde, die Resonanz unserer Medien im Publikum zu messen, sind die Ergebnisse niederschmetternd. Studiert man die Statistik der Arbeitsgemeinschaft Katholische Presse (AKP) in der Bundesrepublik, so zeigt sich ein jährlicher Rückgang, der zwischen zwei und fünf Prozent schwankt. Ein immenser Aderlaß. Davon sind zwar Missionszeitschriften nicht im gleichen Maßstab betroffen wie die Bistumsblätter, aber dies sollte uns nicht zu der Annahme verleiten, sie seien auf Grund ihrer Qualität vom Auflagenschwund verschont. Vielmehr ist zu vermuten, daß hier der Bezug einer Zeitschrift mit einer Missionsspende verwechselt wird.

\section{Mission als „Markenartikel"}

Die Leistungen der kirchlichen Presse und insbesondere der Missionspresse sollen hier nicht unterschlagen werden. Lange bevor die bunten Blätter an den Kiosken ferne Länder und exotische Kulturen entdeckten, hat beispielsweise die Missionspresse über Menschen in fernen Kontinenten und Kulturen informiert. Die Missionspresse hat nicht nur das Dritte-Welt-Bild ganzer Generationen geprägt. Sie hat auch innerkirchlich eine missionarische Spiritualität begründet, die jahrzehntelang den Humus bildete für Missionsberufe und Missionsbegeisterung. Mit der publizistischen Vermarktung der Dritten Welt durch säkulare Medien ist nicht nur das kirchliche Monopol in diesem Bereich gebrochen worden, sondern auch eine Übersättigung eingetreten, die vielfach abträglich ist für ein echtes Engagement.

Neben dem Verlust des publizistischen Monopols in Sachen Dritte Welt, das die Kirche bis in die sechziger Jahre hinein behauptet hatte, droht ihr nun ein zweiter schwerer Einbruch: Die säkularen Medien drängen - teils aus echter Betroffenheit, teils zur eigenen Profilierung - kräftig auf den Spendenmarkt. Der „Tag für Afrika" hat uns vorgeführt, welche Konkurrenz etwa den kirchlichen Hilfswerken ins Haus steht. Sie hätten keinen Grund, über die ungewohnte Sensibilität der säkularen Medien für die Not in aller Welt besorgt zu sein, wenn die Werbekampagnen nicht unmittelbar auf ein kirchliches Publikum ausgerichtet wären. Nicht neue Spendenquellen werden dadurch erschlossen, sondern alte umgeleitet.

Die Missionspresse, die das Thema Mission als Markenartikel vertreibt, muß stärker noch als die herkömmliche Presse gegen eine indifferente bis feindselige Grundstimmung ankämpfen. Schon das Wort "Mission“ ist heute vielfach negativ besetzt, was unter anderem eine Folge der marxistischen Religionskritik wie der anthropologischen Missionskritik ist. Es verwundert aber festzustellen, daß selbst in innerkirchlichen Kreisen Mission häufig als überholt eingestuft wird. Es herrscht ein unbestimmtes Gefühl, daß mit dem Zweiten Vatikanum die Mission als Thema abgetreten sei und höchstens noch in Form von Entwicklungshilfe oder religiösem Dialog eine Daseinsberechtigung 
habe. Unter dem Eindruck der religiösen Renaissance in weiten Teilen der Dritten Welt werden zwar kulturelle und religiöse Faktoren selbst im Kalkül von Entwicklungsstrategen berücksichtigt, ohne daß aber daraus im Westen ein neues missionarisches Bewußtsein entstanden wäre.'

Wenn die Kirche wirklich, wie es das Zweite Vatikanische Konzil betont hat, ihrem Wesen nach missionarisch ist, ist in dieser Charakterisierung der Mission die Chance der Missionspresse aufgezeigt. Die hergebrachten Grenzen der Geographie existieren für die Missionspresse nicht mehr. Sie muß sich mit den Grundfragen der menschlichen Existenz überhaupt beschäftigen. Insofern ist der „Markenartikel Mission“ überaus attraktiv. Die Frage ist nur, ob die kirchliche Publizistik fähig und willens ist, das Thema Mission auch zeitgerecht aufzubereiten.

Mit Sorge ist in diesem Zusammenhang zu beobachten, daß das Niveau der meisten Zeitschriften in den letzten Jahren immer höher geschraubt wurde. Alle Untersuchungen der Leserschaft aber belegen, daß es sich bei den Beziehern der Missionszeitschriften um „kleine Leute“ handelt, also um jene Bevölkerungsschichten in Deutschland, die nach wie vor die wichtigsten Stützen der kirchlichen Arbeit in der Dritten Welt sind. Es ist mittlerweile bekannt, daß es die Armen der Ersten Welt sind, die über die kirchlichen Organisationen die Armen in der Dritten Welt unterstützen. Insofern kommt diesen materiell bescheidenen Bevölkerungsschichten durchaus eine prophetische Rolle zu. Gerade auf diese motivierten Bevölkerungsgruppen konzentriert sich aber immer mehr die „Bildungswut“ der Werke.

In diesem Zusammenhang ist auf das phänomenale Aufsteigen der Regenbogenpresse in den letzten Jahren hinzuweisen. Allein in der Bundesrepublik Deutschland haben sich seit 1980 sieben Wochenblätter in einer Auflagenhöhe um die Millionengrenze etabliert. Es ist bekannt, daß die vorwiegend weiblichen Leser dieser Zeitschriften eine anspruchslose Kost erwartet. Statt allzu eilfertig die Nase darüber zu rümpfen, sollte man - angesichts der Tatsache, daß sich die Leserschaft der Regenbogenpresse weitgehend mit der Leserschaft der Kirchen- und Missionspresse deckt - die Frage stellen, warum jene am Markt verlieren und diese dazugewinnen.

Es hängt vielleicht mit dem verschärften „Bewußtsein“ unserer Publizisten zusammen. Wenn sie das intellektuelle Niveau ihrer Zeitschriften immer höher schrauben, müssen sie auch in Kauf nehmen, daß immer mehr Leser abwandern werden. Gehörten früher zu jedem katholischen Haushalt der Michaelskalender oder die Christliche Familie, trifft man heute eher auf das „Goldene Blatt“ oder die „Frau mit Herz“. Eine offensive Medienstrategie würde bedeuten, daß die Kirche sich mit einer unveränderten Botschaft auf das niedrigste Niveau herunterwagt - übrigens journalistisch eine weitaus größere Herausforderung als die Belieferung mit elitären Fachzeitschriften.

\section{Auf der Suche nach der verlorenen Sprache}

Abschließend bleibt festzuhalten, „Mission und Medien“ bedingen einander, stehen aber heute eher in latenter Spannung als in wechselseitiger Zustimmung. Was die kirchliche Publizistik, das eigentliche Standbein der Kirche in der Öffentlichkeit, betrifft, bedarf es von seiten der "Macher" sicherlich mehr an Professionalität. Von seiten der Herausgeber, die in der Regel mit der sogenannten Amtskirche identisch sind, bedarf es größerer Souveranität und Liberalität. Der restaurative Wind, den viele heute in der Kirche zu verspüren glauben, hat jedenfalls in der kirchlichen Publizistik niemals aufgehört zu wehen. 
Eine Besserung des Gesamtklimas zwischen Kirche und Medien ist nur zu erwarten, wenn die Kirche eine bedeutende Vorleistung bringt. Sie muß ein Stück Inkulturation betreiben. Beim Begriff ,Inkulturation“ denkt man fälschlicherweise nur an die Kontinente Asien und Afrika. Dabei wird übersehen, daß auch in der westlichen Welt die christliche Botschaft in eine nicht-mehr-christliche Kultur hineingetragen werden muß. Die Kirche Europas hat beklagt, daß ihr nacheinander die Arbeiterschaft, die Jugend und zu guter Letzt auch die Frauen abhanden gekommen sind. Sie hat dabei übersehen, daß sie auch die Sprache verloren hat. Unsere Kirche wird lernen müssen, wieder die Sprache ihrer Zeit zu sprechen. Nur dann wird sie ein glaubwürdiger Partner der modernen Kulturträger, zu denen mehr denn je die Medien zählen: Mit einem „Aschermittwoch der Künstler" allein kommt noch kein Gespräch mit einer säkularisierten Kultur zustande. Die fällige „Inkulturation“ der christlichen Botschaft wird jeder Generation neu abverlangt, und die Medien sind dafür unverzichtbare Werkzeuge.

\section{Anmerkung:}

1 Es müßte nachdenklich stimmen, wenn neue, hoffnungsträchtige Initiativen, die sich etwa mit der Umwelt, dem Frieden und den Menschenrechten befassen, sich außerhalb der Kirche ansiedeln (Friedensbewegung, Amnesty International, Greenpeace, Robin Wood, etc.). Selbst unverkennbare Ansätze einer religiösen Erneuerung in der Jugend werden häufig von Bewegungen aufgefangen, die außerhalb der beiden Volkskirchen angesiedelt sind. Oder sie führen zur Bildung von esoterischen Zirkeln und Vereinen, denen es eher um individuelle Spiritualität oder interne Club-Harmonie bestellt ist, als darum, in eine bestehende Pfarrgemeinde, in die Gesellschaft oder die Welt missionarisch hineinzuwirken. 


\section{SUMMARY}

Catholic Church, well knowing how to use the verbal medium according to her interests during the past, today is more or less isolated in the media environment of the Federal Republic of Germany. This situation can be explained partly by her own preoccupation against video, partly by the reluctant behaviour of the editors. According to Bauer, media work in the context of mission, is a substantial part of Church. In recent years, Church engagement was more or less limited to experiments with expensive new technologies. Church trying to economise expenditures for personnel, many of these attempts have failed. Church publications, the author says, do not take into account the linguistic level of the majority of their readers. More and more these drift away to secular media have put the caritative themes of Church press on their agenda.

\section{RÉSUMÉ}

LEglise catholique qui a su au cours de son histoire recourir admirablement á la parole pour défendre ses intérêts, est aujourd'hui absente dans le paysage de la communication sociale en République Fédérale d'Allemagne. Cette situation s'explique d'une part par sa réserve envers les médias et d'autre part par l'attitude négative des rédactions à son égard. La présence de l'Eglise dans les médias devrait faire partie essentielle de son travail missionnaire. Ces dernières années cependant, l'engagement de l'Eglise dans ce domaine s'est épuisé en expériences dans des nouvelles technologies très coûteuses. Par souci d' économie en frais de personnel, beaucoup de ces tentatives ont échoué. Les publications de l'Eglise, dit l'auteur, utilisent un language qui n'est pas accessible à la majorité des lecteurs. Dès lors un nombre de plus en plus important d'entre eux se tourne vers des médias séculiers qui reprennent les thèmes caritatifs de la presse de l'Eglise.

\section{RESUMEN}

En el transcurso de su historia, la Iglesia Católica ha sabido recurrir admirablemente al uso de la palabra para defender sus intereses, sin embargo en la actualidad está prácticamente ausente del contex to de la Comunicación Social en la República Federal Alemana. Esta situación se podría explicar por una parte por su posición reservada hacia los media en general y por otra parte la actitud negativa de las redacciones frente a la Iglesia. La presenncia de la Iglesia en los media debería ser una parte esencial de su trabajo misionero, sin embargo en estos últimos años la participación de la Iglesia en este dominio se ha agotado en la realización de experiencias con nuevas tecnologías muy costosas. A su vez ha habido una preocupación por ahorrar los costos de personal lo que ha conducido al fracaso de muchas de estas iniciativas. Según el autor, las publicaciones de Iglesia no utilizan un lenguaje accesible para la mayoría de sus lectores. En consecuencia, un grupo cada vez más numeroso de lectores se informa de los temas caritativos de la Iglesia a través de los media seculares que difunden este tipo de información. 